\title{
COVID-19 screening in patients with eye emergencies: practical experience from a tertiary eye hospital
}

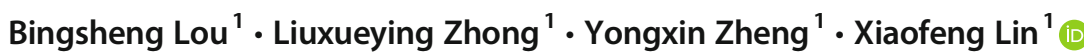 \\ Received: 29 April 2020 / Revised: 28 July 2020 / Accepted: 1 August 2020 / Published online: 14 August 2020 \\ (C) Springer-Verlag GmbH Germany, part of Springer Nature 2020
}

\section{Key messages}

- The methods used to confirm COVID-19 are not suitable for widespread screening in eye hospitals. It is challenged to quickly and effectively treat eye emergency patients while avoiding the transmission of COVID-19.

- Non-contact temperature measurements and epidemiological history inquiry are used for primary screening in all patients with eye emergencies. While in the hospital, the walking path of the patient should be minimised.

- For patients who need surgery, routine blood examination, chest imaging examination, and nucleic acid testing should be performed for further screening. How to arrange the surgery should be based on the degree of the eye emergency and the risk of COVID-19.

\section{Dear Editor,}

The World Health Organization (WHO) has announced that COVID-19 is a global pandemic. Nucleic acid tests of the tears of COVID-19 patients revealed positive results [1], and there have been a few cases of conjunctivitis as the initial

$\overline{\text { Bingsheng Lou and Liuxueying Zhong contributed equally to this work. }}$

This article is part of a topical collection on Perspectives on COVID-19.

Yongxin Zheng

fdhqs@126.com

$\bowtie$ Xiaofeng Lin

linxiaof@mail.sysu.edu.cn

1 State Key Laboratory of Ophthalmology, Zhongshan Ophthalmic Center, Sun Yat-sen University, Guangzhou 510060, Guangdong, China symptom of COVID-19 [2]. During clinical ophthalmology services, healthcare workers have close contact with patients, which increases the risk of transmission of COVID-19. It has been reported in the news that three ophthalmologists in Wuhan have died of COVID-19. The American Academy of Ophthalmology strongly recommends that all ophthalmologists provide only urgent or emergent care [3].

Eye hospitals are the main medical institutions providing services for eye emergencies. However, eye hospitals rarely set up fever clinics and isolation wards to screen and isolate patients with COVID-19. Thus, eye hospitals must quickly and effectively treat emergency patients while avoiding the transmission of COVID-19. It is difficult to quickly identify COVID-19 patients, especially those in the incubation period and with mild or asymptomatic infections [4]. The confirmation of COVID-19 should be made based on the epidemiological history, clinical symptoms, CT scan findings and nucleic acid 
test results. Different from general hospitals [5], the methods used to confirm COVID-19 are not suitable for widespread application in eye hospitals. The Zhongshan Ophthalmic Center (ZOC) of Sun Yat-sen University is one of the largest eye institutions in China. The ZOC has developed special workflows for COVID-19 screening and the management of patients with eye emergencies (Fig. 1). During the COVID-19 epidemic, the ZOC has provided round-the-clock eye emergency services, and no nosocomial infections have occurred among healthcare workers and patients.

Patients should be screened according to the degree of eye emergency and the risk of COVID-19 (Fig. 1). Before entering the hospital, all patients should undergo non-contact temperature measurements and be questioned for any

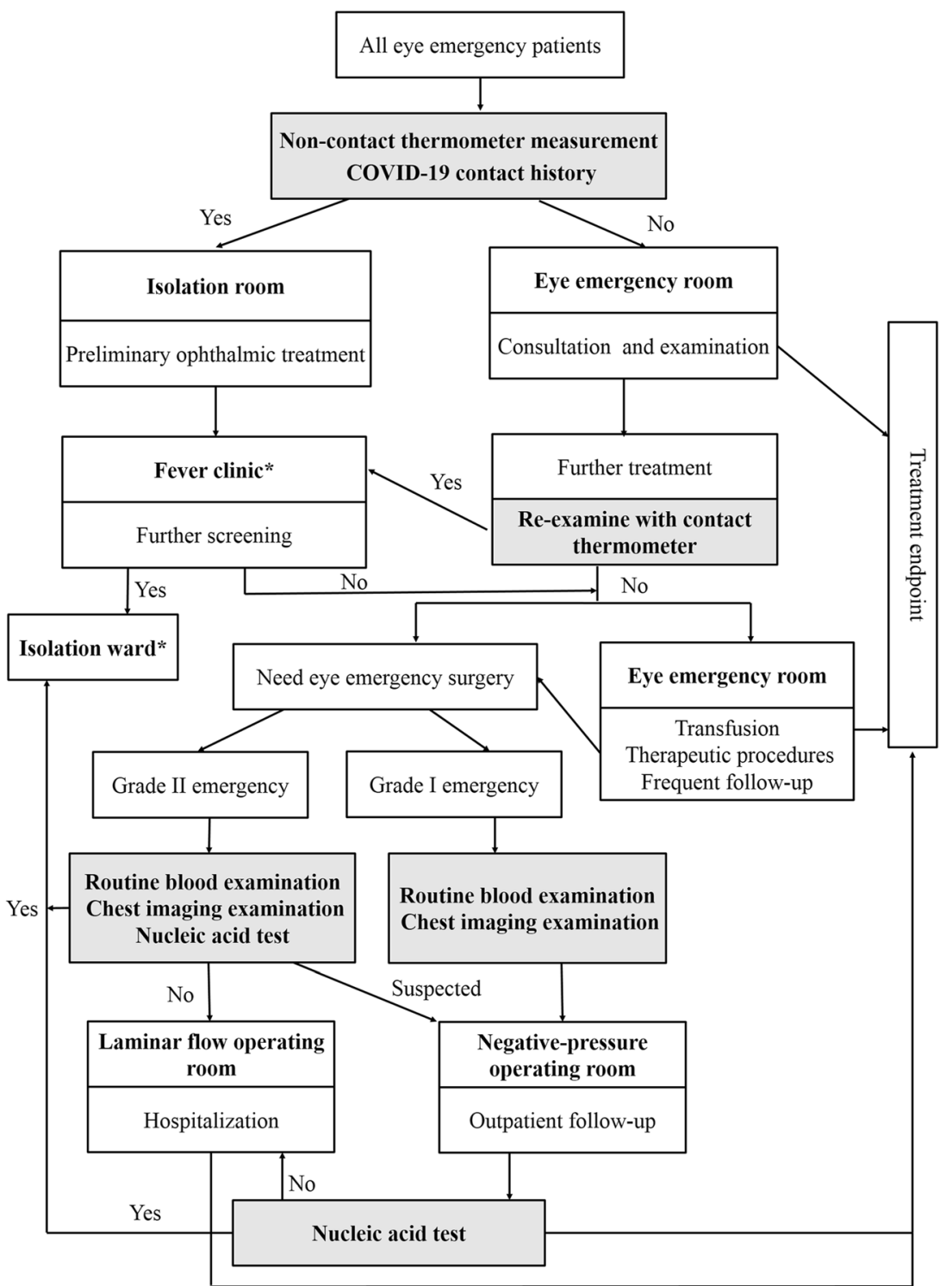

Fig. 1 Workflows for COVID-19 screening and the management of patients with eye emergencies at the ZOC. From January 24, 2020, to March 15, 2020, 6207 patients with eye emergencies were screened at the ZOC. Among them, 132 (2.1\%) patients were found to have fever. A total of $196(3.2 \%)$ patients who needed surgery underwent routine blood examinations, CT imaging examinations and nuclear acid testing. A total of 183 patients underwent surgery at the ZOC (13 patients were referred to the designated general hospital for surgery due to other systemic abnormalities). Open globe injury (32/183 patients), endophthalmitis (14/ 183 patients), eyelid or canaliculi laceration (9/183 patients), corneal ulcer with perforation ( $3 / 183$ patients) and acute angle-closure glaucoma with out-of-control intraocular pressure ( $2 / 183$ patients) were classified as grade I emergencies, while rhegmatogenous retinal detachment (77/183 patients), traumatic retinal detachment (12/183 patients), temporarily controlled acute angle-closure glaucoma (8/183 patients), secondary glaucoma (12/183 patients), ocular malignant tumour (12/183 patients) and intraocular foreign body without endophthalmitis (2/183 patients) were classified as grade II emergencies. A total of 107 (58.5\%) patients were operated on in the laminar flow operating room, and $76(41.5 \%)$ patients were operated on in the negative-pressure operating room. Additionally, 23 patients with suspected lung lesions detected by CT or fever history were followed up for repeated nucleic acid testing. No cases of COVID19 were detected in patients during follow-up. *The fever clinic and isolation ward are located in the designated general hospital near the ZOC 
epidemiological contact history of COVID-19. If fever or any history of epidemiological exposure is detected, then the patients should receive preliminary ophthalmic treatment in an isolation room and be transferred to the fever clinic of the designated hospital for further screening. Even for patients with a normal temperature and without epidemiological history, it is necessary to limit their activities in the hospital. While in the hospital, the walking path of the patient should be minimised. For patients who need to stay for a long time due to advanced treatments (such as transfusion, therapeutic procedures and surgery), a contact thermometer should be used further to re-examine body temperature.

For patients who need surgery, further screening for COVID-19 should be performed. In addition to the indications for conventional emergency surgery, such as open globe injury and endophthalmitis, rhegmatogenous retinal detachment, glaucoma and intraocular foreign body are also treated with emergency surgery at the ZOC due to the cessation of elective surgery during the COVID-19 epidemic peak. All eye emergency surgeries are classified as one of two grades according to the degree of the emergency (Fig. 1). For a grade I emergency, which requires surgical intervention within hours, routine blood examination, chest imaging examination and nucleic acid testing are arranged before surgery, but surgery can be performed without waiting for the results of the nucleic acid test, and it is also permissible to arrange nucleic acid testing after surgery. For grade II emergencies, surgery should be performed after COVID-19 is excluded by all the above test reports. Patients without COVID-19 by the aforementioned examinations are allowed to be admitted to the laminar flow operating room for surgery. For patients for whom COVID-19 cannot be completely excluded, surgery should be performed in the negative-pressure operating room; hospitalisation should be avoided if possible, and a repeated nucleic acid test is obligatory during follow-up. If COVID-19 is confirmed, the patient must be transferred to the isolation ward of the designated hospital immediately.

Furthermore, for patients with a high risk of COVID-19, such as those with fever and acute conjunctivitis, telephone follow-up should be performed. Moreover, healthcare workers must strengthen their personal protection strategies to avoid exposure [6]. Personal protective equipment (PPE), such as surgical masks, goggles, gowns and caps, should be worn throughout the workday. When there is close contact with suspected or confirmed COVID-19 patients, N95 face masks and protection suits are required.

The number of individuals with COVID-19 is still increasing dramatically. This poses a great challenge in treating COVID-19 patients in epidemic areas while maintaining the medical care of patients with non-COVID-19 diseases. Our workflows for COVID-19 screening and the management of patients at the $\mathrm{ZOC}$ are based on the relative balance between COVID-19 epidemic prevention and maintenance of the medical order for eye emergencies. Our experience at the ZOC during the COVID-19 epidemic can provide a reference for providing eye emergency services or gradually restoring ophthalmic non-emergency clinical services in areas with severe COVID-19 outbreaks. Although no nosocomial infections have occurred among healthcare workers and patients at the ZOC thus far, it should be noted that our workflows may not completely block the transmission of COVID-19. Therefore, our experience may not be applicable for all eye hospitals, and it may be necessary to adjust the workflows according to their own medical resource reserves and the severity of the local COVID-19 epidemic.

Funding information This study was supported by the National Natural Science Foundation of China (No. 81760181) and High-Level Hospital Construction Project (No. 303010402).

\section{Compliance with ethical standards}

Conflict of interest The authors declare that they have no conflict of interest.

\section{References}

1. Xia J, Tong J, Liu M, Shen Y, Guo D (2020) Evaluation of coronavirus in tears and conjunctival secretions of patients with SARSCoV-2 infection. J Med Virol. https://doi.org/10.1002/jmv.25725

2. Li JO, Lam DSC, Chen Y, Ting DSW (2020) Novel coronavirus disease 2019 (COVID-19): the importance of recognising possible early ocular manifestation and using protective eyewear. Br J Ophthalmol 104(3):297-298. https://doi.org/10.1136/bjophthalmol2020-315994

3. American Academy of Ophthalmology: New recommendations for urgent and nonurgent patient care (2020) https://www.aao.org/ headline/new-recommendations-urgent-nonurgent-patient-care. Accessed June 262020

4. Bai Y, Yao L, Wei T, Tian F, Jin DY, Chen L, Wang M (2020) Presumed asymptomatic carrier transmission of COVID-19. JAMA. https://doi.org/10.1001/jama.2020.2565

5. Du H, Zhang M, Zhang H, Sun X (2020) Practical experience on emergency ophthalmic surgery during the prevalence of COVID-19. Graefe's archive for clinical and experimental ophthalmology = Albrecht von Graefes Archiv fur klinische und experimentelle Ophthalmologie. https://doi.org/10.1007/s00417-020-04692-x

6. Lai THT, Tang EWH, Chau SKY, Fung KSC, Li KKW (2020) Stepping up infection control measures in ophthalmology during the novel coronavirus outbreak: an experience from Hong Kong. Graefe's archive for clinical and experimental ophthalmology = Albrecht von Graefes Archiv fur klinische und experimentelle Ophthalmologie. https://doi.org/10.1007/s00417-020-04641-8

Publisher's note Springer Nature remains neutral with regard to jurisdictional claims in published maps and institutional affiliations. 